\title{
LA REFORMA DEL SISTEMA ELECTORAL BRITÁNICO: A PROPÓSITO DEL REFERENDUM DEL 5 DE MAYO DE 2011
}

\author{
MARÍA GARROTE DE MARCOS \\ Profesora Titular Interina de Derecho Constitucional \\ Universidad Complutense de Madrid
}

\author{
SUMARIO. \\ I. Introducción. \\ II. El sistema electoral británico. \\ III. Intentos de reforma del sistema electoral. \\ IV. El referéndum sobre reforma electoral. \\ V. Conclusión.
}

\section{INTRODUCCIÓN}

El 5 de mayo de 2011 los ciudadanos británicos fueron convocados a las urnas para decidir en referéndum si deseaban sustituir el vigente sistema electoral de la Cámara de los Comunes - mayoritario simple en distritos uninominales - por el sistema de voto alternativo. Este sistema, que también opera en distritos uninominales, permite al elector expresar sus preferencias entre todos los candidatos y asegura que el diputado sea elegido con la mayoría absoluta de los votos del distrito, ya sea en primeras o sucesivas preferencias. Aunque el sistema de voto alternativo no supone una reforma abrupta del tradicional sistema británico, y desde luego no es un sistema proporcional, consigue eliminar una de las disfunciones que ha sido objeto de mayores reproches: que el candidato pueda conseguir el escaño con un apoyo minoritario en su circunscripción.

El resultado del referéndum ha sido contundente en contra de la reforma propuesta. Pero los antecedentes del proceso y el propio desarrollo político del Reino Unido muestran claramente una nueva etapa en la percepción democrática de su sistema electoral. La evolución del sistema de partidos en Westminster y la progresiva implantación política de partidos distintos al laborista o conservador en otros ámbitos institucionales presagian nuevas iniciativas de reforma, ante la incapacidad del sistema de representar los matices de la cada vez más compleja opinión pública. 
El proceso de convocatoria de referéndum ha venido acompañado de algunas modificaciones muy significativas que afectan a otros elementos del sistema electoral, como el tamaño de la Cámara o el método de delimitación de distritos. Habrá que esperar para ver los efectos políticos de esas reformas en los resultados electorales de los siguientes comicios, pero en el plano jurídico constituyen un formidable avance hacia el principio de igualdad material de la representación.

En estas líneas se pretende abordar someramente este reciente proceso de reformas enmarcándolo en la evolución del sistema electoral británico, que ha sido constante pese a su apariencia de inmovilidad.

\section{EL SISTEMA ELECTORAL BRITÁNICO}

\section{II.1. Evolución DEL SISTEMA ELECTORAL BRITÁNICO: DEL SIGLO XIX HASTA HOY}

El sistema electoral británico es un magnífico ejemplo de adaptación paulatina de las reglas que lo informan a la propia evolución política y social del país.

El reconocimiento del sufragio universal se produjo de forma lenta y progresiva, con sucesivas reformas electorales que ampliaban poco a poco el cuerpo electoral, aunque acompañadas por otras medidas tendentes a atenuar los efectos de dicha ampliación. Hasta el primer tercio del siglo XIX no existía ni una regulación uniforme ni una práctica homogénea en la elección de los representantes en la Cámara de los Comunes. En 1832 cada unidad territorial, county o borough ${ }^{1}$ enviaba dos representes a los Comunes, pero existía una pluralidad de fuentes jurídicas en materia electoral que implicaba una diversidad de criterios en base a los cuales se reconocía el derecho de voto ${ }^{2}$. Con la Reform Act de 1832 se llevó a cabo la primera regulación estatal sobre el derecho de sufragio en todo el país y condujo a un moderado incremento del cuerpo electoral. Las reformas de 1867 y 1884 ampliaron el derecho de voto, aunque en todo caso la titularidad de algún derecho real sobre bienes inmuebles constituía requisito imprescindible para adquirir la condición de elector ${ }^{3}$. En 1884 se introduce también la cualificación por negocio y por pertenencia a la comunidad universitaria (incluyendo graduados) ${ }^{4}$, las cuales podían acumularse y permitían ejercer el voto múltiple.

1 Ambas unidades presentan multitud de divergencias, tanto por su fundación u origen, como por su régimen jurídico. Sin embargo, el principal rasgo diferenciador entre una y otra viene determinado por el sustrato socio-económico: mientras los counties tienen una base esencialmente rural y agrícola, los boroughs son unidades territoriales de carácter comercial y urbano.

2 Sobre la evolución del derecho de sufragio en counties y boroughs, en especial los requisitos de franquicia en ambos, véase el estudio pormenorizado de RUIZ Y RUIZ, J., Evolución del derecho de sufragio en el Reino Unido de la Gran Bretaña e Irlanda, Imprenta de Archivos Olózaga, Madrid, 1929.

3 Esta conexión entre la propiedad y el derecho de sufragio se mantendrá hasta después de la Primera Guerra Mundial, variando en cada momento los criterios de los que depende la cualificación de votante Véase, BOGDANOR, V., The People and the Party System. The referendum and electoral reform in British politics, Cambridge University Press, Londres, 1981; BUTLER, D. E., "The Electoral System in Britain since 1918 », Oxford at the Claredon Press, 1963.; MACKENZIE, K., The English Parliament, Penguin Books, Londres, 1951.

4 Hasta la abolición de este tipo de representación en 1948 la representación universitaria fue variando en número, de 5 a 12 escaños. Véase, REEVE, A., WARE, A., Electoral Systems, Routledge, London, 1.992, p.62. 
Tras la Primera Guerra Mundial, la Representation of the People Act de 1918 instaura definitivamente el sufragio universal masculino sobre la base de tres premisas (residencia, titularidad de un negocio o pertenencia a la comunidad universitaria) y elimina la diferencia de requisitos entre counties y boroughs ${ }^{5}$. Además, limita el ejercicio del voto múltiple a sólo dos y siempre que se ejerciera en distintos distritos. Junto a ello, se produce el primer reconocimiento del derecho de sufragio a las mujeres con ciertas limitaciones, que desaparecerán formalmente en 1928. Esta reforma servirá de punto de partida hacia la transformación de un sistema electoral predemocrático y de elites en un sistema electoral democrático y de masas. En la segunda posguerra la Representation of the People Act de 1948 suprime el ejercicio del voto múltiple, acogiendo manifiestamente el principio de «un hombre, un voto».

En la actualidad, cualquier ciudadano británico, irlandés o de cualquier Estado miembro de la Commonwealth que tenga su residencia en el Reino Unido puede votar en las elecciones parlamentarias, con algunas excepciones, como los miembros de la Cámara de los Lores, los incapaces o los condenados por delitos electorales ${ }^{6}$.

En cuanto al sistema electoral utilizado para la Cámara de los Comunes, se observa una evolución en la utilización de diversas reglas electorales que transcurre paralela a la ampliación progresiva del cuerpo electoral, hasta que en 1950 se adopta definitivamente el sistema mayoritario en distritos uninominales.

Hasta 1832 se elegían dos diputados por cada county o borough con independencia de la población o electorado de cada uno, ya que la representación era estrictamente territorial. Pero con el transcurso del tiempo las desigualdades se hicieron abrumadoras: mientras un buen número de counties se despoblaban drásticamente, las incipientes ciudades pre-industriales como Manchester, Leeds, Sheffield y Birmingham permanecían sin representación propia. Se ha estimado que en 1832 la relación entre el distrito electoral con mayor número de electores y el que contaba con menos era de 100 a $1^{7}$. La reforma electoral de 1832 estableció un nuevo diseño de los distritos electorales, de diversa magnitud según las necesidades de representación ${ }^{8}$. Esta redistribución permitió paliar las anomalías más destacadas pero estuvo muy lejos de significar un intento sistemático de delimitación equitativa de los distritos.

La Reform Act de 1867 también contemplaba una revisión de los límites de los distritos, con el fin de atenuar los efectos de la ampliación del derecho de sufragio. Así, se separaron los counties y antiguos boroughs de las grandes connurbaciones urbanas, pri-

5 No obstante, los distritos electorales siguen clasificándose en la actualidad como counties — rurales_ o boroughs —urbanos_-, lo que afecta a los límites de gastos electorales permitidos para la campaña.

6 Una de los rasgos más peculiares del sistema británico es que permite no sólo el voto por correo — hasta hace poco sometidas a unos severos requisitos - sino que también prevé el voto por poderes, eliminando el carácter personal del voto tan protegido en España. Para un análisis detallado de los titulares del derecho de voto en el Reino Unido y los requisitos para su ejercicio, véase, LEONARD, D.; MORTIMORE, R., Elections in Britain. A voter's guide, Palgrave, Houndmills, 2001, pp. 12-22, así como la bibliografía allí citada.

7 LEONARD, R. L., et al. Elections in Britain, D. Van Nostrand, London, 1968, p.18.

8 Así, 26 counties contaban con cuatro representantes, siete con tres, y Yorkshire con seis. Las ciudades de Birmingham, Leeds y Manchester obtuvieron representación por primera vez, con dos escaños, mientras que Londres tendría cuatro parlamentarios. SPECK, W. A., Historia de Gran Bretaña, Cambridge University Press, Londres, 1996, p. 98. 
mando a los distritos del campo. De esta forma, sólo 200 de los 659 escaños de los Comunes se disputaban en distritos uninominales. Junto a ello, se adoptó el voto limitado en las trece circunscripciones que elegían a tres miembros y en la ciudad de Londres, que disputaba cuatro escaños. Esto suponía que el elector contaba sólo con dos votos o, en su caso, con tres. La introducción del voto limitado respondía a la necesidad de asegurar una justa representación de las minorías frente a la extensión del cuerpo electoral, pero no produjo los efectos previstos. El partido liberal consiguió desarrollar una estrategia para burlar sus efectos y apoderarse de todos los escaños en liza?

Con las reformas de 1885, los dos principales partidos acordaron la eliminación del voto limitado y la generalización de los distritos uninominales, quedando tan sólo 27 circunscripciones con dos escaños, imponiéndose en todo caso el sistema mayoritario simple. Este nuevo diseño redujo las desigualdades entre distritos considerablemente, pero no se previó ningún sistema permanente de revisión, lo que condujo a que las desigualdades afloraran al poco tiempo. Aparte de las diferencias entre distritos, también existía una gran disparidad en la representación otorgada a Inglaterra con respecto a Irlanda y, sobre todo, a Gales y Escocia, notablemente sobrerrepresentadas.

A medida que se va aceptando el principio democrático de «un hombre un voto» la equivalencia de representación entre circunscripciones fue cobrando más importancia. Cualquier reforma en materia electoral debía ir acompañada de una nueva redistribución de los escaños ya que el principio «un hombre, un voto» —one man, one vote — carecía de significado si no iba acompañado del corolario «un voto, un valor» —one vote, one value — ${ }^{10}$. En 1918 se constituyó una Speaker's Conference en el seno de la Cámara para proponer una reforma electoral. Las bases fundamentales iban a ser, aparte de una ampliación del sufragio, la propuesta de introducción de algún tipo de representación proporcional (voto único transferible combinado con el voto alternativo) y una nueva redistribución de los escaños. La adopción del sistema proporcional — como se expondrá más adelantesuscitó enconados debates en el Parlamento y terminó por rechazarse, pero se reconoció el principio de representación proporcional a la población en los distintos distritos. Mediante la creación de una Boundary Commission, encargada de delimitar las circunscripciones de acuerdo con la población de cada una, se procedió a un nuevo diseño del mapa electoral sensiblemente más equilibrado. Las pautas para la delimitación comprendían el respeto a los límites de las administraciones locales y el intento de configurar distritos de tamaño semejante (unos 70.000 electores). Las diferencias entre distritos se redujeron a una proporción de 5 a 1 entre el mayor y el menor y se aproximaron las medias de población por distritos entre Inglaterra, Escocia y Gales. En Irlanda la población media por distrito era sensiblemente inferior, aunque esta disparidad desapareció con la creación del estado Libre de Irlanda en $1922^{11}$. Sin embargo, como no se previó

9 Las victorias liberales en Birmingham o Glasgow se basaron en minuciosos sondeos de opinión seguidos de agresivas campañas electorales aconsejando la disciplina del voto a todos los electores. Véase, BOGDANOR, V., The people... op. cit., pp. 100-104.

10 BUTLER, D.E., op cit, p. 6.

11 Por el contrario, la representación otorgada a Irlanda de Norte en los Comunes de 12 escaños en lugar de los 18 que le corresponderían en proporción a la población, motivó una notable infrarrepresentación de la región. CURTICE, J., «The Electoral System», en BOGDANOR, V. (Ed), The British Constitution in the Twentieth Century, Oxford, Oxford University Press, 2003, p. 497. 
ningún mecanismo de revisión periódica el diseño elaborado en 1918 quedó rápidamente obsoleto. Al final de la Segunda Guerra Mundial los movimientos de población condujeron a severas desigualdades de representación ${ }^{12}$.

En 1944 se aprobó finalmente un sistema permanente de revisión de los límites de los distritos, que serían todos uninominales. La Redistribution of Seats Act de 1944 estableció cuatro Boundary Commissions de composición apolítica, una para cada parte del Reino Unido - Inglaterra, Gales, Escocia e Irlanda del Norte ${ }^{13}$ - que realizarían las revisiones pertinentes en intervalos de entre 3 y 7 años. Las reglas de delimitación partían del cálculo de la cuota electoral, resultado de dividir el número de electores del Reino Unido entre el número total de escaños, que se aconsejaba no incrementar. Para diseñar los distritos era necesario que ninguno se desviara más del $25 \%$ de la cuota y que se respetaran los límites locales, aunque ambas reglas podían ser ignoradas en casos especiales.

Estas pautas parecían dirigirse a asegurar circunscripciones de igual tamaño, pero era incompatible con el compromiso de no disminuir la representación otorgada a Escocia y Gales (71 y 35 escaños respectivamente), pese a que su población no había crecido al mismo ritmo que la inglesa durante el período de entreguerras. Todo ello condujo a que la cuota electoral se calculara separadamente para cada parte del Reino Unido, lo que significaba alejarse del objetivo de distritos de igual tamaño y, sobre todo, del de no incrementar el número de miembros de los Comunes ${ }^{14}$.

En 1948 se aprobó la Revisión Inicial de los distritos realizada por las cuatro Boundary Commissions que se aplicó a las elecciones de $1950^{15}$. De los 625 distritos, sólo 80 permanecieron igual que antes de la revisión. Se procedió a una nueva revisión que fue aprobada en 1955 y que afectó a 251 distritos, además de crear 5 nuevos. Esta delimitación fue muy contestada en el Parlamento por los inconvenientes que provocaba para los partidos y para los diputados la alteración de los límites electorales con tanta frecuencia. Por esta razón, en 1958 se aprobó una reforma de la Ley que ampliaba los plazos de las revisiones a intervalos de entre 10 y 15 años. De esta forma se aprobaron las nuevas redistribuciones en 1974, 1983, 1992 y 2004-2008 ${ }^{16}$. En 1986, la Parliamentary Constituencies Act sistematizó las reglas de redistribución y reguló el procedimiento de revisión con más precisión. Las reglas que deben respetar las Boundary Commissions son cier-

12 En 1944, por mencionar los casos extremos, el distrito de Romford contaba con 220.000 electores, mientras que el Southwark North sólo poseía 14.000.

13 Las Boundary Commissions están formadas en la actualidad por el Speaker de la Cámara de los Comunes — como Presidente nominal—, un Magistrado — que ostenta la Vicepresidencia y en la práctica dirige los trabajos- - y dos expertos independientes propuestos por el Gobierno.

14 Véase, BUTLER, D., op. cit., pp. 92-127.

15 Las recomendaciones de las cuatro BC se publicaron en 1947 y fueron aprobadas por Ley en 1948. Al año siguiente se modificó la Reditribution of Seats Act de 1944 para permitir la relajación de las normas en caso que las BC lo creyeran conveniente. CURTICE, J., «The electoral System», op. cit., p. 498.

16 Normalmente las revisiones periódicas entran en vigor al mismo tiempo en las cuatro partes del Reino Unido, pero en la última revisión la delimitación de los distritos de Escocia se aprobó en 2004 y se pudo aplicar en las elecciones generales de 2005. La revisión de Gales entró en vigor en 2006, la de Inglaterra en 2007 y la Irlanda del Norte en 2008. GAY, O.; WHITE, I., «The Parliamentary Voting System and Constituencies Bill», Research paper 10/55, House of Commons Library, 1 septiembre 2010, pp. 30-31. Se puede consultar en:

http://www.parliament.uk/briefingpapers/commons/lib/research/rp2010/RP10-055.pdf 
tamente pocas y concisas, pero en la práctica son difíciles de conciliar, por las abiertas incompatibilidades que existen entre ellas.

En primer lugar, el número de electores por distrito debe acercarse lo más posible a la cuota electoral. Para ello se debe calcular la cuota de cada parte del Reino Unido separadamente, teniendo en cuenta que Gales y Escocia deben tener un mínimo de 35 y 71 diputados respectivamente, e Irlanda del Norte debe tener 17 escaños (aunque excepcionalmente puede tener 16 ó 18). Esto significa que no hay ningún tipo de límite para Inglaterra, y el número de escaños que se le asigne va a estar condicionado por estos topes. ${ }^{17}$

En segundo lugar, se deben respetar los límites de las administraciones locales en la medida de lo posible.

En tercer lugar, se ofrece la posibilidad de eludir las reglas anteriores si especiales consideraciones geográficas, tamaño, forma o accesibilidad de los distritos, lo hiciesen conveniente. En todo caso, las Boundary Commissions deben tener en cuenta los inconvenientes que se puedan producir con la modificación de los límites de los distritos, especialmente si perturban los vínculos locales.

Es evidente que la aplicación de las dos primeras reglas (respeto a los límites locales y electorado próximo a la cuota) es la cuestión más difícil y controvertida de todo el proceso de delimitación, y su combinación con el resto de criterios produce un incremento constante en el tamaño de la Cámara de los Comunes, que hoy tiene 650 miembros $^{18}$. Junto a ello, la falta de un mecanismo de reparto de escaños entre las distintas partes del Reino Unido, acorde con la población de cada una, produce severas desigualdades entre los electorados de cada una de ellas ${ }^{19}$.

Estas deficiencias en la distribución de la representación se verán suavizadas tras la aprobación de la Parliamentary Voting System and Constituencies Act de febrero de 2011. La ley establece nuevas reglas para la redistribución de escaños, comenzando por una reducción del número de miembros de la Cámara de los Comunes, que se fija en $600^{20}$. El objetivo, además de frenar el constante incremento de parlamentarios, es reducir las desigualdades de electores por distrito. Para ello se va a utilizar una cuota electoral uniforme para todo el Reino Unido, referida al electorado registrado el 1 de diciembre de 2010.

17 Al no existir un límite máximo ni para Gales ni para Escocia, se dota a estas regiones de una representación desmesurada en relación con Inglaterra, pues cualquier aumento en sus escaños, siendo fijo el número de escaños de Gran Bretaña, implica una reducción de representación para Inglaterra. Esta prerrogativa, que se ha venido a denominar «celtic preference», tiene motivaciones poco claras. Véase, MCLEAN. I., «Are Scotland and Wales over-represented in the House of Commons?, The Political Quarterly, $\mathrm{n}^{\circ} 66,1995$, pp. 250-268.

18 Pese a que la ley establece que el número de diputados en la Cámara no debe exceder de 613, la revisión de 1992 condujo a una Cámara de 659 miembros. En la última revisión Escocia ha limitado su representación a 59, como consecuencia de la creación de un Parlamento propio y régimen especial de autonomía mediante la Scotland Act de 1998.

19 Después de la última revisión, y con los datos de las elecciones de 6 de mayo de 2010, el electorado medio de Inglaterra se sitúa en 71.876, mientras que el Gales es de 56.626, el de Escocia 65.526 y el de Irlanda del Norte 64.955. La media del Reino Unido es de 70.170 electores.

20 La ley puede consultarse en http://www.legislation.gov.uk/ukpga/2011/1/contents/enacted.

Una análisis de las nuevas reglas de redistribución en GAY, O.; WHITE, I., «The Parliamentary Voting System...», op.c it., pp. 36-57. 
Los escaños correspondientes a cada parte del reino Unido se asignarán mediante la fórmula Sainte-Laguë. El número de electores por distrito debe ser lo más cercano posible a la cuota electoral y se prohíben las desviaciones superiores o inferiores al 5\% de la cuota. Sólo respetando esta regla se permite tomar en consideración los límites de las administraciones y otros vínculos locales. Asimismo, ningún distrito podrá tener una superficie mayor de $13.000 \mathrm{~km}$ cuadrados (que es tamaño del distrito de Ross, Skye and Lochaber).

La ley prevé también disposiciones especiales para Irlanda del Norte, permitiendo desviaciones superiores de la cuota electoral en esos distritos. Las islas de $\mathrm{Na}$ h-Eileanan An lar y Orkneys and Shetland se mantienen como dos distritos independientes, aunque posean un número de electores muy reducido.

La primera revisión al amparo de estas nuevas reglas se completará el 1 de octubre de 2013 y las siguientes se realizarán cada cinco años, previendo que se aprueben a los tres años del comienzo de cada legislatura. El impacto de las nuevas reglas sobre la próxima revisión es difícil de prever, pero es evidente que conllevará cambios significativos ${ }^{21}$.

\section{II.2. RENDIMIENTOS DEL SISTEMA ELECTORAL BRITÁNICO}

Hoy en día los miembros de la Cámara de los Comunes son elegidos en distritos uninominales con el sistema electoral mayoritario simple. Estos elementos han permanecido invariables desde las elecciones de 1950, pero algunos de los efectos del sistema electoral han evolucionado a medida que se ha ido transformando también el sistema de partidos y el sistema político en su conjunto.

El sistema electoral produce un claro vencedor en las elecciones, que suele obtener una mayoría absoluta de los escaños de la Cámara. Esto facilita la formación de gobiernos fuertes y estables, que tienden a ser más eficaces en las tareas que tienen asignadas. Además, el sistema también permite al electorado castigar al Gobierno con más contundencia, al producir una exageración de los movimientos de opinión en comparación con los sistemas proporcionales. Todo ello mejora el mecanismo de rendición de cuentas y refuerza la responsabilidad de los ejecutivos. Los distritos uninominales favorecen una vinculación más directa y personal del candidato o diputado con el electorado de su circunscripción, al tiempo que les permite ser más independientes de la cúpula de los partidos. Como el que gana, lo gana todo, y el perdedor no se lleva nada, el sistema tiende a excluir las posturas más extremistas — que no pueden obtener representación por sí mismas_-, o al menos obliga a que se incorporen a corrientes más moderadas.

De otro lado, el sistema también implica que los votos dirigidos a los partidos que no resultan vencedores se pierden, de forma que no existe relación entre los votos y los escaños. Los resultados más o menos proporcionales a nivel nacional son absolutamente fortuitos. La sobrerrepresentación del partido ganador de las elecciones, que obtiene siem-

21 Se ha estimado que Inglaterra perderá 30 de los 533 escaños que posee en la actualidad, Escocia 7 de 59, Gales10 de 40 e Irlanda del Norte 3 de 18. La cuota electoral se situará en torno a los 76.180 electores por distrito.

22 Los resultados de las elecciones desde 1950, en porcentajes de votos y escaños se pueden consultar en LEONARD, D.; MORTIMORE, R., Elections in Britain, op. cit., Tabla 4.3, p. 34. 
pre un mayor porcentaje de escaños que de votos, se ha venido explicando con la llamada «ley del cubo». La ventaja de un partido sobre otro en porcentaje de votos se triplica al traducirse en porcentaje de escaños ${ }^{22}$.

No obstante, tras las últimas convocatorias electorales la ley del cubo parece estar en cierto declive, debido principalmente a la existencia de auténticos feudos electorales para conservadores (Sudeste de Inglaterra y zonas rurales) y laboristas (Escocia, norte de Inglaterra y ciudades del interior ${ }^{23}$. La contrapartida es la presencia cada vez más acusada de «desiertos electorales», zonas donde alguno de los dos partidos apenas obtiene representación. Así, uno de los rasgos más característicos de la geografía electoral británica es la progresiva consolidación de distritos seguros — safe seats-para uno u otro partido. Esto deja el espacio político disputable cada vez más exiguo, con sólo unos 150 distritos donde es posible un vuelco electoral — marginal seats-.

El número de escaños que obtiene un partido no sólo depende de los votos que reciba, sino de dónde se reciben esos votos. Ciertos estudios han destacado la interacción entre la implantación geográfica de un partido y la delimitación de los distritos electorales. Los resultados electorales presentan desviaciones y no sólo referidas a la desproporcionalidad contra los terceros partidos, sino en el sentido de que el mismo porcentaje de votos entre los dos mayores partidos se puede traducir en drásticas diferencias en escaños ${ }^{24}$.

Durante los años 50 y 60 los mayores beneficiados de estas desviaciones fueron los conservadores. Entre 1966 y 1987 no hubo desviaciones significativas, pero a partir de 1992 y sobre todo en 2001 y 2005 el sistema benefició notablemente a los laboristas, hasta el punto de que hoy en día se reconoce que sistema favorece abiertamente a este partido. Las razones de estas desviaciones obedecen a distintos factores, pero quizá los más importantes son: los defectos en la delimitación de los distritos, los distintos niveles de participación y el apoyo a un tercer partido, fundamentalmente al Liberal Demócrata.

Pese a que las Boundary Commissions llevan a cabo las delimitaciones de los distritos con imparcialidad, lo cierto es que sus resultados tienen efectos políticos de alcance. Esto se debe a que los laboristas suelen obtener buenos resultados en las ciudades del interior, que están en proceso de despoblación. Por otra parte, la sobrerrepresentación de Escocia y Gales también les beneficia, pues allí son el partido más fuerte. Ambas circunstancias les permiten obtener el máximo rendimiento de sus votos y explican por qué con cada

23 En las últimas elecciones generales celebradas en mayo de 2010, el partido conservador obtuvo un $36.1 \%$ de los votos y $47 \%$ de los escaños, mientras que el partido laborista obtuvo el $29 \%$ de los votos y $36.7 \%$ de los escaños. Los siete puntos de ventaja en votos sólo se han traducido en algo más de 10 puntos en escaños.

24 Véase, ROSSITER, D., et al, «Changing biases in the operation of the UK's Electoral System, 19501997», British Journal of Politics and International Relations, vol. 1, n 2, junio, 1999, pp. 133-164. Puede consultarse en: http://onlinelibrary.wiley.com/doi/10.1111/1467-856X.00008/abstract; JOHNSTON, R., et al., «Distorsion Magnified: New Labour and the British Electoral System, 1950-2001», en BENNIE, L.; RAWLINGS, C.; TONGE, J.; WEBB, P. (Eds.), British Elections and Parties Review, vol. 12: The 2001 General Election, London, Frank Cass, 2002, pp. 133-155.

25 Por ejemplo, en las elecciones de 2005, las últimas celebradas antes de la quinta revisión de distritos, el distrito medio ganado por los laboristas tenía 65.748 electores, mientras que el distrito medio de los con- 
nueva revisión efectuada por las Boundary Commissions, los laboristas suelen perder escaños ${ }^{25}$.

Por otra parte, los feudos electorales de los laboristas suelen ser distritos con baja participación, lo que significa que disminuye el número de votos necesarios para obtener el escaño y ello redunda en la relación votos-escaños. En el mismo sentido, la presencia de los liberales favorece a los laboristas en la medida en que se fomenta el voto táctico anticonservador.

En definitiva, durante los últimos años se ha venido constatando que un mismo número de votos a favor de la partido laborista se traduce en más escaños que la misma cantidad a favor de los conservadores.

La combinación de todos estos efectos, la ley del cubo y las desviaciones electorales ha provocado en ocasiones resultados aberrantes, es decir, que el partido que gana en escaños no haya ganado en votos. Esto se produjo en 1951 y en 1974 . Además, cerca del $40 \%$ de los escaños se consiguen en distritos en los que el candidato no recibe la mayoría absoluta de los votos, porcentaje que va en aumento a medida que obtiene más apoyo un tercer partido (el liberal y los nacionalistas en Gales y Escocia). Esto conduce a una gran paradoja: ¿cómo sostener la representatividad de un parlamentario cuando más de la mitad de los electores han votado contra él?

En conclusión, hoy en día el sistema mayoritario simple en el Reino Unido no produce los efectos políticos que tradicionalmente se han señalado como paradigmáticos. Hay una tendencia a gobiernos con menos mayorías; el sistema de partidos ya no es un two-party-system; desde luego no lo es desde el punto de vista de partidos electorales, pero tampoco respecto a partidos parlamentarios ${ }^{26}$. Las desviaciones que produce y la existencia de feudos electorales provocan cada vez más rechazo en una ciudadanía que ya ha tenido la oportunidad de experimentar con otros sistemas electorales.

\section{INTENTOS DE REFORMA DEL SISTEMA ELECTORAL}

A medida que se iba ampliando el derecho de sufragio y la democracia británica comenzaba a adquirir los rasgos de una democracia de masas fueron surgiendo propuestas de modificación del sistema electoral. Así, la Speaker's Conference de 1917 redactó un Proyecto de Reforma - Representation of the People Bill_ que contenía varias recomendacio-

servadores era de 72.140 electores. En otras palabras, los distritos que ganaron los laboristas contenían un 9\% menos de electores que los ganados por los conservadores. En este sentido, LEONARD, D.; MORTIMORE, R., op. cit., pp. 30-32; MITCHELL, P., «The United Kingdom: Plurality Rule under Siege», en GALLAGHER, M.; MITCHELL, P. (Eds.), The Politics of Electoral Systems, Oxford, Oxford University Press, 2005, pp. 162-163.

26 En las elecciones de 2001 el 12\% de los miembros de la Cámara (80 diputados) no pertenecían a los dos principales partidos; En 2005 ascendió al 14\% de los miembros de la Cámara (92 diputados) y en las celebradas en mayo de 2010 fueron 13,5\% (87 diputados). Considerando la penalización del sistema a las terceras fuerzas políticas, esa presencia parlamentaria muestra una apoyo electoral bastante significativo (en torno al $30 \%$ del voto popular).

27 El Voto único transferible utiliza circunscripciones plurinominales y los votantes marcan a los candidatos en su orden de preferencias. La atribución de escaños se realiza mediante recuentos sucesivos. Después de 
nes, entre ellas la introducción del sistema proporcional conocido como voto único transferible en los boroughs ${ }^{27}$ y el voto alternativo en el resto de distritos ${ }^{28}$.

La debilidad de la propuesta provenía de su propio planteamiento: carecía de justificación que el sistema proporcional sólo se aplicase en un tercio de las circunscripciones. De hecho, sólo fue defendida por los parlamentarios que no iban a ser elegidos por esas circunscripciones, mientras que los que iban a verse afectados por el cambio se oponían totalmente. En todo caso, los partidos permitieron libertad de voto a sus parlamentarios y el resultado fue un forcejeo entre los Comunes y los Lores. Los Comunes rechazaron la propuesta por cinco veces, sustituyéndola por el voto alternativo generalizado, mientras que los Lores intentaron por dos veces introducirlo de nuevo, rechazando el voto alternativo, pensando que éste perjudicaría notablemente a los conservadores porque tendía a aglutinar el voto de izquierda. ${ }^{29}$

Finalmente se terminó aceptando el sistema mayoritario simple por puro desistimiento, aunque la falta de acuerdo y la polémica suscitada en torno al asunto provocó una solución de compromiso, ya que se pensaba que continuar con los debates indefinidamente podía traer consigo el fracaso de todo el Proyecto. La única concesión al sistema proporcional se hizo respecto a las circunscripciones universitarias donde se terminó implantando el sistema de voto único transferible en cuatro de ellas ${ }^{30}$.

En cualquier caso, ningún partido tenía una posición definida respecto al tema, dentro de las mismas filas conservadoras y liberales había defensores y opositores. El único partido que votó unido y siempre en el mismo sentido (a favor) fue el partido nacionalista irlandés

A partir de entonces la representación proporcional estuvo vinculada al partido liberal, que acabó siendo el principal perjudicado del sistema mayoritario cuando el partido laborista

contar el número total de primeras preferencias, se utiliza una operación para establecer la cuota electoral —número de votos requeridos para la elección de un candidato- Se atribuyen los escaños a los candidatos que reúnan un número de primeras preferencias igual o superior a la cuota. Si ninguno logra la cuota, el candidato con el menor número de primeras preferencias es eliminado y sus segundas y terceras preferencias son redistribuidas entre los candidatos restantes. Al mismo tiempo, el «excedente» de votos de los candidatos elegidos (en relación a la cuota) es redistribuido de acuerdo a las segundas preferencias de las papeletas. El procedimiento de nuevas transferencias y recuentos continúa hasta la adjudicación de todos los escaños. Sobre el sistema de voto único transferible y su funcionamiento en la República de Irlanda, véase, VALLÉS, J.M., BOSCH, A., Sistemas electorales y gobierno representativo, Ariel, Barcelona, 1997, pp. 235-240

28 El voto alternativo o preferencial es un medio para favorecer la formación de mayorías absolutas, ya que reúne en una sola operación el mecanismo del sistema mayoritario a dos vueltas. El votante indica en primer lugar el nombre de su candidato preferido y, a continuación, señala un orden de prioridad entre los demás candidatos presentados. Si un candidato obtiene la mayoría absoluta de las primeras preferencias, es proclamado vencedor. Si ninguno la obtiene, se eliminan a los candidatos con menos votos de primera opción, pasando dichos votos a segundas o terceras opciones. Se repite el procedimiento hasta que un candidato consigue reunir un número de preferencias equivalentes a la mayoría absoluta. Sobre el sistema de voto alternativo y su funcionamiento en Australia, véase VALLÉS, J.M., BOSCH, A., op. cit., p.88 y 217 y ss.

29 Las votaciones en los Comunes fueron muy reñidas. En dos ocasiones se rechazó el sistema proporcional por apenas un voto. Sin embargo, las diferencias entre los que apoyaban la propuesta y los que se oponían a ella fueron aumentando votación tras votación para terminar con el rechazo de la misma por 169 votos contra 112. Véase BOGDANOR, V., op cit., p.128 y ss, y BUTLER, D.E., op cit., p. 11 y ss.

30 CURTICE, J., «The Electoral System», op. cit., p. 505.

31 El Congreso del Partido Laborista que siguió al Informe recomendó la adopción del voto suplementario, una modalidad del voto alternativo que permite al elector indicar sólo dos preferencias. Véase, NORRIS, 
se afianzó como alternativa a los conservadores. Cuando tras las elecciones de 1929 ningún partido obtuvo la mayoría, se pensó que la causa de esta situación era el sistema electoral. Se constituyó una Comisión con representación de todos los partidos que recomendó la adopción de algún tipo de representación proporcional, aunque sin mucho éxito. En 1930 el Gobierno laborista presentó un Proyecto de Reforma Electoral que suponía la adopción del voto alternativo. El proyecto fue aprobado en primera lectura con el apoyo de los liberales, pero en la Cámara de los Lores se aprobó una enmienda que reducía la implantación del voto alternativo a sólo un tercio de los distritos (Londres y los grandes boroughs). De este modo, se produjo un conflicto entre ambas Cámaras que condujo a la paralización del Proyecto, hasta que la caída del Gobierno en agosto de 1931 provocó su retirada definitiva.

A medida que se iba afianzando el sistema bipartidista y las elecciones producían mayorías absolutas de gobierno, la cuestión de la reforma electoral fue perdiendo interés. Pero en 1974, tras dos elecciones consecutivas en las que ninguna fuerza política obtuvo la mayoría absoluta y el voto a los terceros partidos alcanzó niveles semejantes a los de 1929, el debate sobre la reforma volvió a resurgir, aunque no se tradujo en iniciativas legislativas concretas.

El impulso definitivo a las reformas provino del partido laborista, tras cuatro derrotas consecutivas entre 1979 y 1992. El partido creó una Comisión dirigida por Lord Plant para examinar la cuestión. Pese a no alcanzar ningún acuerdo sobre cuál podría ser el sistema electoral alternativo, el debate suscitado en el seno del partido laborista persuadió a su líder para convocar un referéndum sobre reforma electoral ${ }^{31}$. Este compromiso se hizo oficial cuando el partido accedió al Gobierno en 1997. Como paso previo, en diciembre del mismo año se creó una Comisión Independiente que tenía como principal tarea analizar las posibles alternativas al sistema electoral vigente en el Reino Unido. El Gobierno señaló los requisitos que debía reunir el sistema propuesto, que no eran precisamente compatibles entre sí: que permitiera una amplia proporcionalidad en los resultados; que facilitara gobiernos estables; que aumentara la capacidad de elección de los votantes; y que mantuviera el vínculo entre los candidatos y los electores.

El Informe que remitió la Comisión al Parlamento contenía una serie de recomendaciones $^{32}$, pero se inclinaba a favor de introducir un sistema de elección mixto, que conjugara dos niveles territoriales y dos métodos de adjudicación de escaños. Así, el 80-85\% de los escaños de la Cámara se elegirían en circunscripciones uninominales, mientras que el resto serían elegidos en listas presentadas en ámbitos territoriales más amplios (top-up areas). Estas circunscripciones, que coincidirían con los grandes counties y áreas metropolitanas, recogerían los votos restantes en los distritos uninominales una vez adjudicados los escaños en ellos. En total se estimaban unas 65 circunscripciones de lista en Inglaterra, cinco en Gales, seis en Escocia y dos en Irlanda del Norte. Para garantizar un

P., «The politics of electoral reform in Britain», en International Political Science Review, vol. 16, $\mathrm{n}^{\circ} 1$, enero 1995, Londres, Sage Publications Ltd., pp. 65-78,

32 Véase, The Report of the Independent Commission on the Voting System, 2 vols, The Stationery Office, Londres, 1998.

También se puede consultar en: www.archive.official-documents.co.uk/document/cm40/4090/4090.htm

33 Véase la Scotland Act de 1998 (http://www.legislation.gov.uk/ukpga/1998/46/contents) y la Goverment of Wales Act de 1998 (http://www.legislation.gov.uk/ukpga/1998/38/contents) 
mayor margen de elección a los votantes también se aconsejaba la introducción del voto alternativo en los distritos uninominales y listas abiertas en las circunscripciones.

Parece que la solución propuesta por la Comisión Independiente no convenció al Gobierno, que no llegó a convocar el referéndum previsto. Sin embargo, durante esa legislatura se acometieron varias reformas en el sistema electoral de otras instituciones, que claramente tendían a una mayor proporcionalidad. Así, en el Parlamento escocés y en la Asamblea de Gales se implantó un sistema electoral mixto, semejante al propuesto por la Comisión independiente, pero sin la utilización del voto alternativo en los distritos uninominales y con listas cerradas y bloqueadas en las circunscripciones ${ }^{33}$. En la Asamblea de Irlanda del Norte se adoptó el sistema de voto único transferible, que ya se venía utilizando en las elecciones locales ${ }^{34}$. En 1999 se reformó la ley que regulaba las elecciones al Parlamento Europeo y se impuso el sistema D'Hondt con listas cerradas y bloqueadas $^{35}$. Por ultimo, en 2000 se creó la Greater London Authority, con una asamblea que es elegida también por el mismo sistema mixto utilizado en Gales y Escocia ${ }^{36}$.

En su programa electoral de 2005, el partido laborista adquiría el compromiso de revisar la experiencia de los nuevos sistemas electorales implantados recientemente y continuaba afirmando que el referéndum era el único camino posible para la reforma del sistema electoral de los Comunes. El Informe sobre los rendimientos de los distintos sistemas fue publicado en $2008^{37}$. El Gobierno puso de relieve las implicaciones que tendría la adopción de un sistema proporcional en Westminster, sobre todo la presencia de pequeños partidos en la Cámara, la tendencia a gobiernos de coalición y la necesidad de configurar circunscripciones plurinominales. La objeción más importante se refería a los gobiernos de coalición, por el cambio que suponía en la usual formación y desarrollo del gobierno en el Reino Unido.

No obstante, al final de la legislatura se presentó un proyecto de ley (Constitutional Reform and Governance Bill 2009-10) que, entre otras cuestiones, establecía las reglas necesarias para celebrar un referéndum sobre reforma electoral para introducir el voto alternativo en la elección de los diputados. Al final de la tramitación, y ante la inminente disolución de la Cámara, las disposiciones relativas a la celebración del referéndum fueron suprimidas ${ }^{38}$. A pesar de ello, lo cierto es que existía una clara predisposición política para abordar un cambio en el sistema electoral y esta cuestión iba a ser intensamente tratada durante la campaña electoral de 2010. Por primera vez en ochenta años la reforma electoral era una realidad políticamente insoslayable.

34 Véase la Northern Ireland Act de 1998 http://www.legislation.gov.uk/ukpga/1998/47/contents

35 La European parliamentary elections Act de 1999 fue reformada en 2002 (http://www.legislation.gov.uk/ukpga/2002/24/contents)

36 Véase la Greater London Authority Act de 1999 (http://www.legislation.gov.uk/ukpga/1999/29/contents). Mediante la Local Goverment Act de 2000 se introdujo el sistema de voto suplementario para la elección del Alcalde (http://www.legislation.gov.uk/ukpga/2000/22/contents)

37 Review of Voting Systems: the experience of new voting systems in the United Kingdom since 1997. Cm 7304, enero 2008. Puede consultarse en: http://www.justice.gov.uk/publications/docs/voting-systems-review-full.pdf

38 Durante el llamado proceso de «wash-up» previo a la disolución de la Cámara, el Gobierno mantuvo negociaciones con los conservadores para sacar adelante la ley y evitar su caducidad, pero tuvo que renunciar a algunos aspectos. Véase, Constitutional Reform and Governance Bill 2008-09 to 2009-10, http://services.parliament.uk/bills/2009-10/constitutionalreformandgovernance.html

39 Véase, GAY, O., «Regulation for the Alternative Vote referendum», Standard Note 5142, House of Commons Library, 28 febrero 2011. Se puede consultar en: http://www.parliament.uk/briefing-papers/SN05142 


\section{EL REFERÉNDUM SOBRE REFORMA ELECTORAL}

Aunque en el Reino Unido no existen normas constitucionales que exijan la convocatoria de un referéndum para aprobar reformas de alcance constitucional, se han celebrado algunos, casi todos de ámbito territorial limitado. En 1975 se celebró un referéndum nacional para la continuidad del Reino Unido en la entonces Comunidad Económica Europea; en 1973 y en 1979 se celebraron sendos referéndums en Irlanda del Norte y en Escocia y Gales para aprobar un especial régimen de autonomía. Durante la legislatura de 1997-2001 se celebraron referéndums en Escocia, Gales y el Greater London para confirmar el régimen de descentralización de esas regiones. En 1998 se convocó un referéndum en Irlanda del Norte para ratificar los «Acuerdos de Viernes Santo». Todos ellos eran referéndums pre-legislativos, pues no se había desarrollado aún la legislación necesaria para regular las cuestiones consultadas.

En el año 2000 se aprobó la Political Parties, Elections and Referendum Act (en adelante, PPERA), que regula, entre otras materias, las competencias que posee la Electoral Commission - la Administración electoral británica, en adelante EC — para organizar y supervisar el desarrollo de los referéndums ${ }^{39}$. En resumen, la PPERA establece lo siguiente: La EC debe establecer un período de campaña electoral durante la cual se controlarán los gastos generados por las organizaciones y grupos participantes; examinará la redacción de la pregunta que es objeto de consulta.; puede designar a «organizaciones paraguas» (umbrella organisations) que gestionen las campañas del «sí» y «no» y que cuentan con subvenciones y ayudas estatales (envíos postales de propaganda gratuitos, elaboración de encuestas, etc), pero están sometidas a unos límites de gastos ${ }^{40}$; durante al menos un período de 28 días antes de la celebración del referéndum la publicidad institucional y gubernamental es objeto de restricciones; y, por último, el Presidente de la EC será el responsable del desarrollo del referéndum.

\section{IV.1. ANTECEDENTES}

Desde que estallaron los escándalos de corrupción política y malversación de fondos públicos del año 2009 se ha venido insistiendo desde todos los ámbitos en la necesidad de una regeneración democrática del país. Una de las propuestas más recurrentes es de la de reducir el número de miembros de la Cámara de los Comunes para recortar gastos y modificar el sistema de elección de los parlamentarios ${ }^{41}$.

El programa electoral del partido conservador para las elecciones generales de mayo de 2010 incluía la reducción del tamaño de la Cámara en un 10\%, así como una modificación de las normas que regulan la delimitación de los distritos, con el fin de asegurar

$40 \mathrm{La}$ existencia de estas «umbrella organisations» resulta algo extraña al sistema español, donde la campaña electoral, pero sobre todo la financiación de la misma, está vinculada exclusivamente a los partidos políticos

41 En la actualidad el número de diputados es de 650 (533 de Inglaterra, 18 de Irlanda del Norte, 59 de Escocia y 40 de Gales).

42 El proyecto se presentó a la Cámara el 22 de julio de 2010. 
una representación más equitativa. El partido liberal demócrata, por su parte, defendió la adopción del sistema de voto único transferible en circunscripciones plurinominales y reducir la Cámara a 500 miembros. Los laboristas abogaban por la introducción del sistema de voto alternativo.

Tras las elecciones la coalición gubernamental formada por conservadores y liberales acordó presentar la Parlamento un proyecto de ley para convocar un referéndum sobre reforma electoral, con el objeto de reemplazar el actual sistema mayoritario simple por el sistema de voto alternativo. Además, en al misma norma se regulaba la reducción del número de diputados, quedándose la Cámara con 600 y unas nuevas reglas para delimitar los distritos electorales ${ }^{42}$.

El proyecto no estuvo precedido de ningún tipo de estudio acerca de las diferentes alternativas al sistema electoral en vigor o sobre reglas de delimitación de distritos, lo que unido a su perentoria tramitación provocó severos reproches de la oposición laborista $^{43}$.

Cuando el Viceprimer Ministro del Gobierno explicó los puntos clave del proyecto anunció que el referéndum se celebraría el 5 de mayo de 2011, en coincidencia con otras elecciones (las elecciones locales en Inglaterra e Irlanda del Norte, las elecciones a las Asambleas de Gales e Irlanda del Norte y al parlamento de Escocia). Junto a ello, advirtió que bastaría que la mayoría simple de los votantes en la consulta apoyaran la reforma para que se diera por ratificada, sin necesidad de un umbral mínimo de participación electoral. Ambas cuestiones suscitaron abundantes críticas.

Respecto a la coincidencia de varias consultas el mismo día, se manifestaron dos preocupaciones: en primer lugar, las notables diferencias de participación que se podrían dar a lo largo y ancho del país, dependiendo de si el referéndum iba a acompañado o no de otras elecciones $^{44}$; y en segundo lugar, la coincidencia podría invitar a la confusión de los electores ${ }^{45}$.

La EC en una declaración emitida el 22 de julio de 2010 manifestó que era posible esa coincidencia de consultas, siempre que las normas relativas al referéndum estuvieran claramente establecidas con al menos seis meses de antelación a la celebración el mismo. Sin embargo, en 2002 la propia EC desaconsejó unir el referéndum sobre el Euro con las elecciones regionales de Escocia, Gales e Irlanda del Norte, porque las elecciones con marcado carácter partidista podrían influir en una consulta que no debería tener dicho carácter ${ }^{46}$.

43 Además, no consideraban adecuado reunir en al misma ley cuestiones tan diversas y políticamente relevantes como el referéndum y la nueva distribución de escaños. Véase, GAY, O.; WHITE, I., «The Parliamentary Voting System...», op. cit., pp. 4-5.

44 El referéndum iba a coincidir con otras elecciones de uno u otro tipo en todo el Reino Unido, salvo en Londres.

45 En 2007 en Escocia hubo una gran cantidad de votos nulos porque se combinaron las elecciones al parlamento escocés y las locales, con dos sistemas electorales distintos, uno de los cuales, el voto único transferible, se utilizaba por primera vez.

46 GAY, O.; WHITE, I., «The Parliamentary Voting System...», op. cit., p. 9. En España está expresamente prohibido la coincidencia de un referéndum con otro tipo de elecciones, salvo los previstos en el procedimiento de reforma constitucional (art. 4.2 Ley Orgánica 2/1980, de 18 de enero, sobre Regulación de las Distintas Modalidades de Referéndum). 
El requerimiento de una mayoría cualificada de electores que se pronuncien a favor para aprobar el referéndum responde a la necesidad de asegurar la suficiente legitimación y aceptación del resultado. En el Reino Unido no existen previsiones al respecto, pero en el referéndum celebrado en 1979 se incluyó el requisito de que al menos el $40 \%$ de los ciudadanos con derecho a voto aceptasen la propuesta para que ésta saliera adelante, siempre que los votos negativos no superaran los positivos.

Muchos parlamentarios estaban a favor de incluir un umbral mínimo pero el Gobierno lo rechazó argumentando que éste podría convertirse en un perverso incentivo para no participar, pues la abstención equivaldría a un voto negativo. Por lo tanto, el resultado del referéndum se computaría sobre los votos emitidos, sin mínimo exigido.

\section{IV.2. LA REGULACIÓN DEL REFERÉNDUM}

La Parliamentary Voting System and Constituencies Act de 2011 regula dos cuestiones diferentes aunque con cierta conexión entre ellas. En primer lugar, detalla las reglas a seguir para la convocatoria y celebración de un referéndum sobre reforma electoral en el Reino Unido. Dicho referéndum se centra en la sustitución del actual sistema mayoritario simple en distritos uninominales por otro conocido como Voto Alternativo. Este sistema también se desenvuelve en distritos uninominales, pero difiere en lo que se conoce como modalidad de voto, otorgando al elector la posibilidad de expresar sus preferencias por los candidatos presentados, y exige la mayoría absoluta de los votos de los electores en primeras o sucesivas preferencias.

Por otra parte la ley también dispone la reducción de los miembros de la Cámara de los Comunes de los 646 actuales a 600, magnitud que permanecerá invariable en adelante. En relación con ello, se modifican sustancialmente las reglas que informan la labor de las Boundary Comissions para permitir una representación equitativa de todos los distritos, como se ha analizado en las páginas precedentes. La consecuencia de todo ello es que a partir de ahora el número de diputados correspondientes a cada parte de Reino Unido no estará sometido a límites mínimos o máximos, sino que será el que le corresponda por población, eliminado una de las causas más importantes de las desigualdades hoy existentes.

En caso de aceptarse la reforma electoral, el nuevo sistema de elección no se aplicaría hasta que no se aprobara la revisión de los límites de los distritos efectuada al amparo de las nuevas normas, y que está prevista para octubre de 2013. Sin embargo, las disposiciones relativas a la delimitación de distritos entrarán en vigor en todo caso.

La ley determina las normas que se verían afectadas en caso de aprobarse la reforma electoral y su alcance, y permite que se implemente el nuevo sistema por Decreto.

Dentro de las cuestiones relativas al desarrollo del referéndum, destacaremos lo siguientes puntos:

Según la ley, tendrán derecho a votar en el referéndum aquellos ciudadanos que lo ostenten para las elecciones parlamentarias, incluyendo también a los miembros de la Cámara de los Lores.

De acuerdo con lo previsto en la PPERA, la pregunta que se formularía en el referéndum fue remitida a la EC para su examen de inteligibilidad. La EC, tras un estudio basado 
en técnicas cualitativas de investigación, propuso una redacción alternativa ${ }^{47}$. La pregunta inicial era: "Do you want the United Kingdom to adopt the «Alternative Vote» system instead of the current «First Past The Post» system for electing Members of parliament to the House of Commons?»

Los requisitos exigidos por la EC para la inteligibilidad son: que la pregunta debe ser accesible a todos los electores, centrada, factual y neutral. A la vista de los resultados del estudio se aconsejó cambiar la estructura del enunciado, dividiéndolo en dos frases cortas y modificar algunos términos ${ }^{48}$, de forma que la pregunta que finalmente se ha sometido a referéndum ha sido: "At the present, the UK uses the "first past the post» system to elect MPs to the House of Commons. Should the "alternative vote» system be used instead?"

En todo caso, el estudio reveló que la mayor parte de los ciudadanos no conocían en absoluto el funcionamiento del voto alternativo, ni tampoco los efectos que podría tener en el plano político, por lo que se insistió en la necesidad de promover una amplia campaña informativa para instruir al electorado. Por supuesto, esta campaña correría a cargo de la propia EC, que en marzo de 2011 comenzó a difundir casi 28 millones de folletos informativos por todo el país, y emitió anuncios en los medios de comunicación.

La ley también prevé la aplicación de la PPERA de 2000 para la regulación de los gastos electorales y sus límites, así como las disposiciones aplicables en caso de coincidencia de otras elecciones el mismo día del referéndum.

\section{IV.3. LA CAMPAÑA ELECTORAL}

La ley establece que el período de campaña electoral comienza con la sanción real de la misma y requiere, según la PPERA un mínimo de diez semanas de duración. La ley fue sancionada el 16 de febrero de 2011.

El 18 de febrero el Primer Ministro David Cameron anunció que el partido conservador defendería el «no» en el referéndum, por considerar que era una reforma absolutamente equivocada, excesivamente compleja, injusta, poco clara e inexplicable.

Por su parte, el Viceprimer Ministro declaró el apoyo de su partido a la reforma propuesta, basándose en los aspectos más críticos del sistema vigente ${ }^{49}$.

Esta discrepancia en el seno del Gobierno de coalición podría ser contradictoria con el principio de responsabilidad colectiva del Gobierno, aunque existen antecedentes semejantes ${ }^{50}$.

La EC designó el 18 de marzo de 2011 las organizaciones que gestionarían ambas opciones en la campaña. «Yes in may 2011 LTD» (promovida por la plataforma «Yes for

47 La EC encargó dicho estudio a una empresa especializada, Define Research \& Insight. Véase, «Voting System for electing MPs to the House of Commons: Referendum question Testing». Qualitative research report for the Electoral Commission, Septiembre 2010. disponible en: http://www.electoralcommission.org. uk/_data/assets/pdf_file/0006/102687/Electoral-reform-question-testing-full-report-website.pdf

48 Las razones para modificar el enunciado de la pregunta fueron diversas, principalmente que no cumplía el requisito de accesibilidad a todos los electores, por el lenguaje poco familiar, y que no era considerada totalmente objetiva y neutral. Véase, «Voting system for electing MPs...», op. cit. Pp. 65-66.

49 Ambas declaraciones en, «Cameron and Clegg announce their very alternative views on vote reform», Times, 19 febrero 2011.

50 El más notorio fue en 1975, con ocasión del referéndum para la continuidad del Reino Unido en al CEE, pero en este caso la división afectaba a miembros del mismo partido, el laborista. 
fairier votes») dirigiría la campaña del «sí», mientras que «No campaign LTD» (promovida por No to $A V$ ) asumiría la defensa del «no».

Durante la campaña, los argumentos que ofrecieron los contrarios a la reforma fueron básicamente: 1) El sistema de voto alternativo es tremendamente costoso de implantar (se calculaba un gasto cercano a los 250 millones de libras); 2) Es complejo e injusto. El candidato situado en segundo o tercer lugar, puede ser el elegido; 3) Sólo se utiliza en tres países (Australia, Fidji y Papua Nueva Guinea); 4) Es un «arreglo» de los políticos, que conduce a Parlamentos débiles y negociaciones en la sombra, de forma que en lugar de ser los electores quienes designen al Gobierno, serán los propios políticos.

Por su parte, los partidarios de la reforma alegaban: 1) Que los diputados trabajarán más y mejor para ganarse el puesto o para poder permanecer en él; 2) El nuevo sistema otorga más capacidad de elección a los votantes que, al ordenar sus preferencias, consiguen que aunque su candidato favorito no gane puedan seguir teniendo influencia en los resultados; 3) Al aumentar la competitividad se eliminarán los distritos seguros — safe seats-y con ello se acabarán los cargos «para toda la vida».

En realidad, las consecuencias políticas del cambio no estaban del todo claras. Aunque se realizaron estimaciones de los posibles resultados que se obtendrían con el sistema de voto alternativo, lo cierto es que era un ejercicio arriesgado pues los efectos de la reforma no sólo serían de tipo mecánico sino también psicológico y, por tanto, muy difíciles de prever. Por una parte, es posible que al incrementar las posibilidades de elección las primeras preferencias se dirigieran a partidos minoritarios, como ocurre en otro tipo de elecciones $^{51}$. De esta forma la proporcionalidad entre las primeras preferencias recibidas por los partidos y los escaños obtenidos sería aún menor que con el sistema vigente. Por otra parte, parece que el sistema favorecería a los laboristas y liberales, pero resulta complicado aventurar a qué partido irían a parar las segundas preferencias de los electores ${ }^{52}$.

\section{IV.4. LOS RESULTADOS}

El referéndum celebrado el 5 de mayo de 2011 arrojó los siguientes resultados ${ }^{53}$ :

La participación fue del $42 \%$ del electorado (en cifras absolutas, 19,2 millones de los 45,7 millones de electores con derecho a voto). La participación más alta, superior al $50 \%$, se registró en Irlanda del Norte y Escocia, donde el referéndum coincidía con las elecciones a los respectivos parlamentos autónomos. La participación más baja se dio el Londres $(35,3 \%)$, donde no coincidía con otros comicios.

51 En las elecciones de 2010 sólo el 10\% de los votantes apoyaron a un partido distinto al conservador, laborista o liberal; pero en las Europeas de 2009 ese porcentaje ascendió a 43\%. HIX, S.; JOHNSTON, R.; MCLEAN, I., «Electoral reform. A Vote for Change?, en Political Insight, Vol. 1, n 2, septiembre 2010, p. 62.

52 En las elecciones de mayo de 2010, los liberales ocuparon el tercer puesto, después de conservadores y laboristas, en 286 distritos. Los laboristas lo ocuparon en 206 y los conservadores en sólo 95. Estos datos permiten deducir que en base a las segundas preferencias laboristas y liberales podrían conseguir el escaño en muchos de esos distritos. HIX, S.; JOHNSTON, R.; MCLEAN, I., «Electoral reform...», op. cit., p. 63.

53 Los resultados detallados pueden consultarse en:

http://ukreferendumresults.aboutmyvote.co.uk/en/default.aspx 
Se emitieron 6,2 millones de votos a favor del cambio $(32,1 \%)$ y 13 millones de votos en contra $(67,9 \%)$. El voto negativo fue mayoritario en todas las regiones del Reino Unido, y superó el $70 \%$ en cinco de las nueve regiones de Inglaterra (North East, West Midlands, East Midlands, East of England y South East). Las regiones con mayor proporción de votos a favor fueron Irlanda del Norte $(43,7)$ y Londres $(39,5)$

De las 440 áreas de recuento, el voto negativo fue mayoritario en 430 . Las 10 áreas en las que venció el «sí» eran seis de Londres, dos de Escocia (Edimburgo y Glasgow), Cambridge y Oxford. En todas estas áreas el gobierno local correspondiente está en manos del partido liberal, laborista o el nacionalista escocés, y los diputados de las mismas provienen mayoritariamente de esos partidos ${ }^{54}$.

De hecho, según las encuestas, el $71 \%$ de los votantes liberales apoyaban la reforma, así como el $41 \%$ de los votantes laboristas. Sólo el 12\% de los votantes conservadores votaría «sí» en el referéndum. Entre los votantes que apoyaban la reforma, la mayoría consideraba que el nuevo sistema proporcionaría unos resultados más justos, que impediría que los candidatos fuesen elegidos con menos de la mitad de los votos del distrito y que podría ser un primer paso hacia la adopción de un sistema proporcional en el futuro.

Entre los votantes contrarios a la reforma, las razones fundamentales de su decisión eran que el sistema vigente es simple, más claro y directo que los demás y que se ha probado que funciona razonablemente bien en el Reino Unido.

\section{CONCLUSIÓN}

Aunque el resultado del referéndum haya impedido un cambio en el sistema electoral británico, lo cierto es que la experiencia ha constituido un importante precedente para posteriores iniciativas, que seguramente no tardarán en surgir.

No es casualidad que en dos legislaturas consecutivas se haya planteado la reforma del sistema electoral con iniciativas legislativas precisas y directas provenientes de gobiernos de distinto color político. No sólo ha sido gracias a la presión que ejerce el partido liberal, por su ubicación en el centro del espectro político y su carácter de partido bisagra, sino también porque muchos sectores académicos, sociales y políticos llevan reclamando la reforma o al menos el replanteamiento del sistema vigente desde años atrás.

Es posible que el punto de inflexión lo haya marcado la introducción de sistemas electorales más o menos proporcionales en otros ámbitos institucionales que han permitido a los ciudadanos comprobar las diferencias entre unos y otros ${ }^{55}$.

El resultado negativo del referéndum estaba previsto desde el momento en que el principal partido sostenedor del Gobierno proclamara su postura abiertamente en contra de la propuesta. La estrategia política del partido liberal para conseguir un cambio se ha visto

54 MCGUINNESS, F.; HARDACRE, J., «Alternative Vote Referendum 2011. Analysis of results», Research paper 11/44, House of Commons Library, 19 mayo 2011, pp. 12-15. Puede consultarse en: http://www.parliament.uk/briefing-papers/RP11-44

55 Inglaterra, exceptuando Londres, es la única parte del Reino Unido que no utiliza otros sistemas electorales distintos al mayoritario simple, salvo para las elecciones europeas. ¿Tiene esto alguna relación con el hecho de que haya sido allí precisamente donde el rechazo a la reforma haya sido más contundente? 
muy debilitada, pero quizá en otra coyuntura política pueda tener más éxito. Sin embargo, el proceso de reforma es un trayecto largo y difícil, en el que las fuerzas políticas deben valorar su capacidad de respuesta, en términos de intereses, resultados y actitudes políticas ${ }^{56}$

El sistema político británico afronta una etapa de revisión de algunas de sus instituciones $^{57}$ y de cambio en el modelo político-constitucional tradicional ${ }^{58}$. El sistema de partidos también está experimentando una transformación a la que necesariamente se le tiene que dar respuesta desde las instituciones. El modelo de democracia británica de tipo mayoritario parece debilitarse y abrirse paso hacia un modelo más consociativo. Nada de esto puede ser ajeno al debate sobre la reforma electoral de la Cámara de los Comunes y permite asegurar que la cuestión no ha quedado definitivamente zanjada.

$$
* * *
$$

TITLE: The British electoral system reform: overview of the referendum of the 5th may, 2011.

RESUMEN: Tras las elecciones generales de mayo de 2010, el Gobierno de coalición formado por conservadores y liberales decidió convocar un referéndum el 5 de mayo de 2011 para reformar el vigente sistema electoral. Para ello, la Parliamentary voting system and Constituencies Act 2011, regula con detalle la introducción del sistema de Voto Alternativo para la Cámara de los Comunes, en caso de que el resultado del referéndum fuera positivo, pero también incluye otras modificaciones de gran trascendencia. Aunque el $68 \%$ de los votantes en el referéndum rechazaron la reforma del actual sistema mayoritario, la experiencia ha constituido un importante precedente para posteriores iniciativas, que seguramente no tardarán en surgir. Esta reseña comienza repasando la evolución del sistema electoral británico hasta la actualidad, para analizar después el desarrollo del proceso de referéndum, sus antecedentes y connotaciones políticas.

ABSTRACT: After 2010 General Elections, the coalition government formed by conservatives and liberals decided to bold a referendum on May 5, 2011 on reforming the current electoral system. For this, the Parliamentary Voting System and Constituencies Act 2011 made provision for the introduction of the Alternative Vote for the House of Commons, in case the referendum result was positive, but also includes other changes of great significance. Although $68 \%$ of voters in the referendum rejected changing the present single-member plurality system, the experience was an important precedent for future initiatives, which probably will soon emerge. This overview begins by reviewing the evolution of the British electoral system to the present, and then analyzes the development of the referendum process, its background and political connotations.

Palabras Clave: Reforma electoral. Referéndum. Cámara de los Comunes.

KEYWORDS: Electoral reform. Referendum. House of Commons.

FECHA DE RECEPCIÓN: 16.06.2011 FeCHA DE ACEPTACIÓN: 29.07.2011

56 Como pone de manifiesto Adrian Blau, la existencia de un Gobierno en minoría o de un Parlamento débil no basta para culminar una reforma electoral. Ello puede ser el acicate para iniciarla, pero si se inicia puede no aprobarse, y si se aprueba puede que no llegue a implantarse, pues requiere al menos dos legislaturas, en las quizá el Gobierno ya no esté en minoría. Véase, BLAU, A., «Majoritarianism under pressure: The electoral and Party Systems», en HAZELL, R. (Ed.), Constitutional Futures Revisited. Britain Constitution to 2020, Londres, Palgrave Mcmillan, 2008, pp. 233-248.

57 Durante la campaña de las elecciones generales de 2010 todos los partidos plantearon la necesidad de reformar la Cámara de los Lores en una Cámara electiva. El Gobierno de coalición se ha comprometido a crear un Comité que prepare las propuestas que se incluirán a un futuro proyecto de ley. HIX, S.; JOHNSTON, R.; MCLEAN, I., «Electoral Reform...», op. cit., p. 66.

$58 \mathrm{El}$ proceso de descentralización política ha sido determinante en este sentido, pero también la revisión del poder judicial y el papel cada vez más relevante de instituciones independientes políticamente. Véase HAZELL, R. (Ed.), Constitutional Futures Revisited. Britain Constitution to 2020, Londres, Palgrave Mcmillan, 2008. 
